\title{
Resolution Enhancement of Diffusion-Weighted Images by Local Fiber Profiling
}

\author{
Pew-Thian Yap and Dinggang Shen \\ Department of Radiology and Biomedical Research Imaging Center (BRIC) \\ The University of North Carolina at Chapel Hill, U.S.A. \\ \{ptyap, dgshen\}@med.unc. edu
}

\begin{abstract}
Diffusion-weighted imaging (DWI), while giving rich information about brain circuitry, is often limited by insufficient spatial resolution and low signal-to-noise ratio (SNR). This paper describes an algorithm that will increase the resolution of DW images beyond the scan resolution, allowing for a closer investigation of fiber structures and more accurate assessment of brain connectivity. The algorithm is capable of generating a dense vector-valued field, consisting of diffusion data associated with the full set of diffusion-sensitizing gradients. The fundamental premise is that, to best preserve information, interpolation should always be performed along fiber streamlines. To achieve this, at each spatial location, we probe neighboring voxels in various directions to gather diffusion information for data reconstruction. Based on the fiber orientation distribution (FOD), directions that are more likely to be traversed by fibers will be given greater weights during interpolation and vice versa. This ensures that data reconstruction is only contributed by diffusion data coming from fibers that are aligned with a specific direction. This approach respects local fiber structures and prevents blurring resulting from averaging of data from significantly misaligned fibers. Evaluations suggest that this algorithm yields results with significantly less blocking artifacts, greater smoothness in anatomical structures, and markedly improved structural visibility.
\end{abstract}

\section{Introduction}

Due to the nature of diffusion magnetic resonance imaging, acquiring images higher than the typical $2 \mathrm{~mm}$ isotropic resolution is extremely difficult without incurring unrealistic scan times and causing very low SNR due to reduced voxel size. Increasing the resolution, however, is not only important for registration, segmentation, and tractography to be performed with greater accuracy, but is also crucial for better visualization of anatomical structures to identify possible neuropathologies. Solutions to achieve higher resolution include employing higher magnetic fields or stronger/faster gradients, dedicated acquisition techniques [4, 8], as well as post-processing algorithms [1,6]. In this paper, we will take the last approach and demonstrate that resolution such as $(1 \mathrm{~mm})^{3}$ can be achieved purely via post-processing techniques. 
A decent super-resolution algorithm called Track-Density Imaging (TDI) was recently proposed by Calamante et al. [1]. TDI gains its image contrast by computing the number of fiber streamlines traversing each element of a highresolution image grid. In order to generate a super-resolution image, whole-brain probabilistic tractography is first performed (generated by randomly seeding a large number of tracks throughout the brain, e.g., $>1,000,000)$. From these fiber tracks, the total number of tracks present in each grid element is then calculated. While promising, TDI is limited for the following reasons: 1) The results can only be as accurate as the tractography algorithm employed; 2) Contrast can only be extracted from regions that can be reached by the fiber streamlines (i.e., regions with reasonably high diffusion anisotropy); and 3) Only scalar maps, computed from fiber statistics, can be generated.

In this paper, we present a technique that will exploit the continuity information given by local fiber structures to increase the resolution of DWI data beyond the initial scan resolution. Our algorithm will generate a spatially dense vector-valued field consisting of diffusion data associated with the full set of diffusion-sensitizing gradient directions. Very much in the spirit of TDI, our approach gains spatial resolution by using additional information obtained from outside each individual voxel. Our approach, however, has a slightly different aim with the following important distinctions:

1. Directional Profiling - Instead of relying on tractography, our approach uses a directional profiling scheme to study the neighborhood of each spatial location and gauge the probability of whether a specific direction is likely to be traversed by fibers. The directional probability distribution is employed to encourage interpolation along the tangential and not orthogonal directions of fiber streamlines. Unlike the conventional trilinear interpolation approach, which has no concern of the directional structure of DWI data, our approach mimics DWI acquisition mechanism more closely by infusing information from different directions to reconstruct the DWI data at each spatial location.

2. Microstructure-Preserving Smoothing - Smoothed diffusion data allow fiber pathways to be tracked more reliably and in a continuous manner. Equally important is the preservation of boundaries that defines the spatial extent of individual structures. In contrast to many existing methods that use inter-voxel gradient information to constrain smoothing to relatively homogeneous regions, our method uses intra-voxel fiber orientation distributions (FODs) to guide smoothing. By constraining interpolation along fiber streamlines, we not only preserve boundaries of the white matter, but also those between fiber tracts within the white matter.

3. Complete DWI Data - Our approach generates a vector-valued field of DWI data corresponding to the full set of diffusion-gradients and is not limited to the white matter. Gray matter provides contextual information for the white matter and the availability of gray matter data allows tissue 
segmentation based on diffusion data, such as that done in [5], to be performed, providing complementary tissue contrast to tissue segmentation based on structural MRI.

\section{Approach}

To increase resolution, the image domain is divided using a grid with grid elements that are smaller than the acquired voxel size. The DWI data for each of these grid elements is then reconstructed using the following steps: 1) Directional profiling in a field of FODs [2] 2) Interpolation of DWI data based on the FOD profile; and 3) Bias correction associated with the Rician distribution nature of the magnitude signal. Each step is detailed in the following sections.

\subsection{Local Fiber Profiling}

To determine the probability of whether a grid element at spatial location $\mathbf{x}$ is traversed by fibers in direction $\mathbf{v}_{k}(k=1, \ldots, M)$, we profile the field of FODs $\{p(\mathbf{x}, \mathbf{v})\}$ in the neighborhood of $\mathbf{x}$ along direction $\mathbf{v}_{k}$ (see Fig. 1). The FOD profile is defined as:

$$
\hat{p}\left(\mathbf{x}, \mathbf{v}_{k}\right)=\frac{\sum_{\mathbf{x}_{i} \in \mathcal{N}\left(\mathbf{x}, \mathbf{v}_{k}\right)} w\left(\mathbf{x}_{i}, \mathbf{x}, \mathbf{v}_{k}\right) p\left(\mathbf{x}_{i}, \mathbf{v}_{k}\right)}{\sum_{\mathbf{x}_{i} \in \mathcal{N}\left(\mathbf{x}, \mathbf{v}_{k}\right)} w\left(\mathbf{x}_{i}, \mathbf{x}, \mathbf{v}_{k}\right)}
$$

where $\mathcal{N}\left(\mathbf{x}, \mathbf{v}_{k}\right)$ is a neighborhood of voxels in the vicinity of a reference line radiating from $\mathbf{x}$ in the direction $\mathbf{v}_{k}$. The weights are determined by

$$
w\left(\mathbf{x}_{i}, \mathbf{x}, \mathbf{v}_{k}\right)=\exp \left(-\frac{d_{\text {axial }}^{2}\left(\mathbf{x}_{i}, \mathbf{x}, \mathbf{v}_{k}\right)}{2 \sigma_{\text {axial }}^{2}}\right) \exp \left(-\frac{d_{\text {radial }}^{2}\left(\mathbf{x}_{i}, \mathbf{x}, \mathbf{v}_{k}\right)}{2 \sigma_{\text {radial }}^{2}}\right)
$$

where $d_{\text {axial }}$ and $d_{\text {radial }}$ are respectively the axial distance (length parallel to the reference line) and the radial distance (length perpendicular to the reference line), as illustrated in Fig. 1]. Specifically,

$$
d_{\text {axial }}\left(\mathbf{x}_{i}, \mathbf{x}, \mathbf{v}_{k}\right)=\left(\mathbf{x}_{i}-\mathbf{x}\right) \cdot \mathbf{v}_{k}
$$

and

$$
d_{\text {radial }}\left(\mathbf{x}_{i}, \mathbf{x}, \mathbf{v}_{k}\right)=\left\|\mathbf{x}_{i}-\mathbf{x}-d_{\text {axial }}\left(\mathbf{x}_{i}, \mathbf{x}, \mathbf{v}_{k}\right) \mathbf{v}_{k}\right\|
$$

where $\|\cdot\|$ is the Euclidean norm. Parameters $\sigma_{\text {axial }}$ and $\sigma_{\text {radial }}$ control the falloff of the weight with respect to the axial and radial distances, respectively. The resulting FOD profile $\hat{p}\left(\mathbf{x}, \mathbf{v}_{k}\right)$ is a directional function that allows for anisotropic interpolation of neighboring information to reconstruct the DWI data of the grid element at location $\mathbf{x}$. 


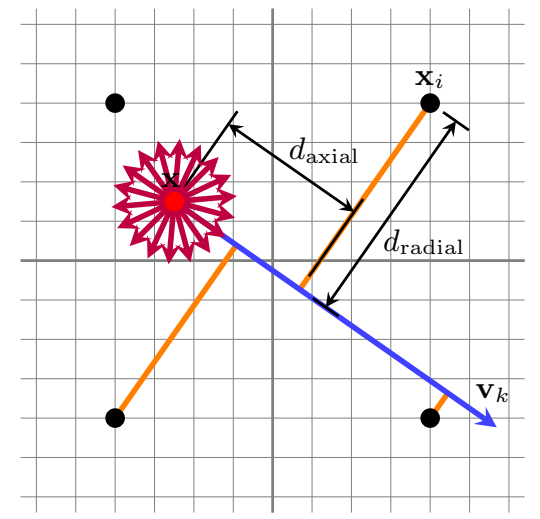

Fig. 1. Directional Profiling. The space resided by the voxels (black dots) are divided by a grid with resolution finer than the scan resolution. For each grid element of interest (red dot), we probe its neighborhood in directions $\mathbf{v}_{k}, k=1, \ldots, M$ (red arrows) to obtain a FOD profile $\hat{p}\left(\mathbf{x}, \mathbf{v}_{k}\right)$, which is essentially a directional function informing us of the certainty of interpolating in a certain direction. The location $\mathbf{x}$ and direction $\mathbf{v}_{k}$ defines a reference line. Voxels closer to the reference line (blue), measured with $d_{\text {axial }}\left(\mathbf{x}_{i}, \mathbf{x}, \mathbf{v}_{k}\right)$ and $d_{\text {radial }}\left(\mathbf{x}_{i}, \mathbf{x}, \mathbf{v}_{k}\right)$, will be given greater weights for interpolation.

\subsection{Fiber-Sensitive Interpolation with Rician-Bias Correction}

To reconstruct the DWI data at location $\mathbf{x}$, interpolation is performed with the help of the FOD profile:

$$
\hat{S}\left(\mathbf{x}, \mathbf{g}_{l}\right)=\left[\frac{\sum_{k} \hat{p}\left(\mathbf{x}, \mathbf{v}_{k}\right) R\left(\mathbf{x}, \mathbf{v}_{k}, \mathbf{g}_{l}\right)}{\sum_{k} \hat{p}\left(\mathbf{x}, \mathbf{v}_{k}\right)}-2 \sigma_{\text {rician }}^{2}\right]_{+}^{\frac{1}{2}}
$$

where

$$
[z]_{+}= \begin{cases}z, & z>0 \\ 0, & \text { otherwise }\end{cases}
$$

and

$$
R\left(\mathbf{x}, \mathbf{v}_{k}, \mathbf{g}_{l}\right)=\frac{\sum_{\mathbf{x}_{i} \in \mathcal{N}\left(\mathbf{x}, \mathbf{v}_{k}\right)} w\left(\mathbf{x}_{i}, \mathbf{x}, \mathbf{v}_{k}\right) S^{2}\left(\mathbf{x}_{i}, \mathbf{g}_{l}\right)}{\sum_{\mathbf{x}_{i} \in \mathcal{N}\left(\mathbf{x}, \mathbf{v}_{k}\right)} w\left(\mathbf{x}_{i}, \mathbf{x}, \mathbf{v}_{k}\right)} .
$$

To avoid blurring of the reconstructed data by data in directions that have low probability of being traversed by fibers, we consider in (5) only directions with $\left.\hat{p}\left(\mathbf{x}, \mathbf{v}_{k}\right)\right\rangle\left\langle\hat{p}\left(\mathbf{x}, \mathbf{v}_{k}\right)\right\rangle_{\mathbf{v}_{k}}$. Note that the squared signal values are used here so that the statistical bias $2 \sigma_{\text {rician }}^{2}$ can be removed for unbiased estimation. This is derived from the fact the second order moment of a Rician distributed quantity is given as $E\left(\mathbf{S}^{2}\right)=S_{\text {true }}^{2}+2 \sigma_{\text {rician }}^{2}$, where $S_{\text {true }}$ is the true signal value. The noise variance $\sigma_{\text {rician }}^{2}$ associated with the Rician distribution [7] can be estimated from the background signal $\left(S_{\text {true }}=0\right)$ using $\sigma_{\text {rician }}^{2}=\sqrt{\left\langle S_{\text {background }}^{2}\right\rangle / 2}$. 


\section{Experimental Results}

We evaluated the effectiveness of the proposed method using a set of in vivo DWI data. For all cases, we set $\sigma_{\text {axial }}=2$ and $\sigma_{\text {radial }}=1$. Directional profiling was performed in 642 directions, which were generated by subdividing the faces of an icosahedron 3 times.

\subsection{Dataset}

Diffusion-weighted images for 4 adult subjects were acquired using a Siemens 3T TIM Trio MR Scanner with an EPI sequence. Diffusion gradients were applied in 120 non-collinear directions with diffusion weighting $b=2000 \mathrm{~s} / \mathrm{mm}^{2}$, flip angle $=90^{\circ}$, repetition time $(\mathrm{TR})=12,400 \mathrm{~ms}$, and echo time $(\mathrm{TE})=116 \mathrm{~ms}$. The imaging matrix was $128 \times 128$ with a rectangular FOV of $256 \times 256 \mathrm{~mm}^{2}$. The slice thickness was $2 \mathrm{~mm}$. $T_{1}$-weighted structural images were also acquired as anatomical references.

\subsection{Computation of the FOD Field}

The FOD field was computed by fitting mixtures of tensors to the DWI data. An over-complete set of tensors were used and the fitting problem was solved using an $L_{1}$-constrained sparse representation framework [2]. We utilized an active-set-based algorithm that was modified from the feature-sign algorithm presented in [3] to incorporate a non-negative constraint. This approach to FOD estimation is known to have great robustness to noise.

\subsection{Methods for Comparison}

We up-sampled each DW image from its original resolution of $(2 \mathrm{~mm})^{3}$ to $(1 \mathrm{~mm})^{3}$. The results given by the standard trilinear interpolation, performed both on the anisotropy map as well as the vector-valued DWI data, were shown for comparison. The generalized anisotropy at each voxel location was computed as the standard deviation to RMS ratio of the signal values.

\subsection{Qualitative Evaluation}

A set of representative results are shown in Fig. 2 together with close-up views in Fig. 3. It is clear from the results that the proposed method yields results that exhibit significantly less blocking artifacts, greater smoothness in anatomical structures, and markedly improved structural visibility. Note that interpolation of DWI benefits quite significantly from interpolation schemes that consider the directionality of the data. From the figure we can see that when interpolation is performed on the scalar anisotropy image, the resulting image is blurred with not much structural details. Details are however much more readily visible when interpolation is performed on the vector-valued DWI data, i.e., by independently 


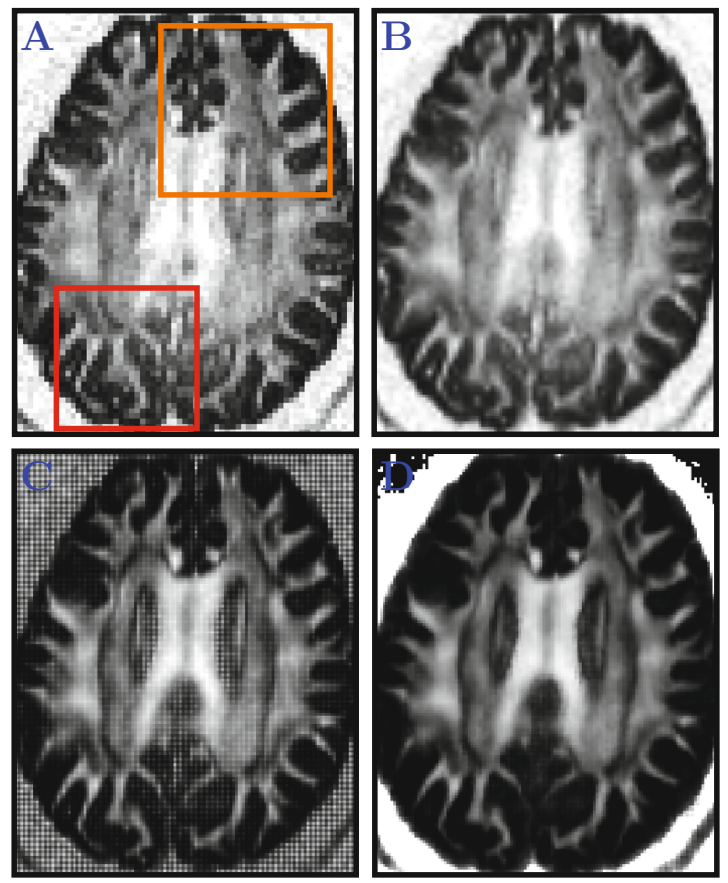

Fig. 2. DWI Resolution Enhancement. DW images were up-sampled to an $(1 \mathrm{~mm})^{3}$ resolution from the original $(2 \mathrm{~mm})^{3}$ resolution. (A) The anisotropy image of the original data; (B) The linearly up-sampled anisotropy image; (C) The anisotropy image of the linearly up-sampled DWI data; and (D) The anisotropy image given by the proposed method. Close-up views of the regions marked in orange and red are shown in Fig. 3

interpolating each element of the data vector across space. This is largely due to the fact that by doing so we are essentially computing a convex combination of neighboring diffusion data that is associated with each particular gradient direction. While clearly giving more structural details, vector-based linear interpolation results in significant blockiness due to the Cartesian nature of the interpolation. All these problems are effectively dealt with using the proposed method, as is evident from the results.

\subsection{Quantitative Evaluation}

For quantitative evaluation, we computed the local variances of the generalized anisotropy values in the white matter (WM), gray matter (GM) and cerebrospinal fluid (CSF). For this purpose, we segmented the $T_{1}$-weighted image associated with each of the 4 subjects into WM, GM, and CSF. These segmented images were then used as masks to compute the average anisotropy variances for regions corresponding to WM, GM, and CSF. The variability of the anisotropy values gives us an indication on how smooth the interpolated image is. The 

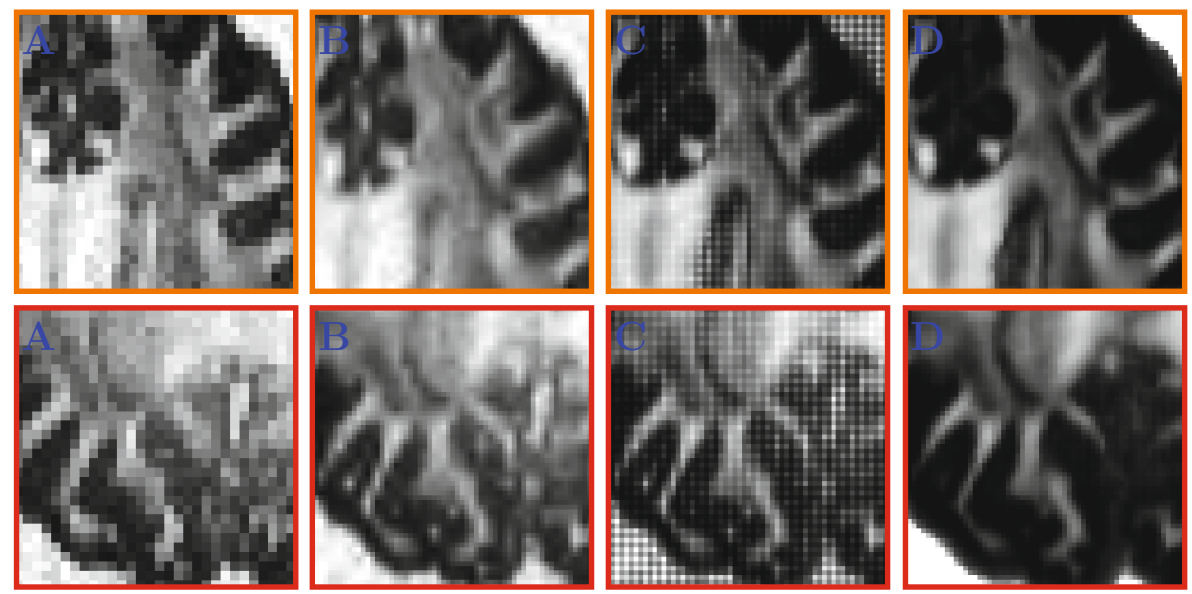

Fig. 3. Closeup Views. Regional closeup views of Fig. 2.
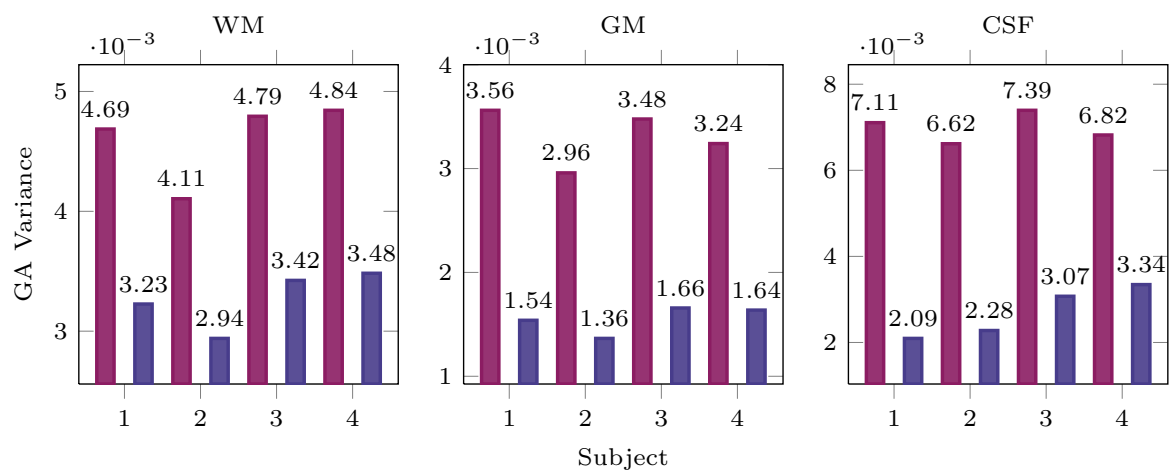

Fig. 4. Average Regional Variance of Generalized Anisotropy. The anisotropy variance at each voxel location is computed using a $3 \times 3 \times 3$ neighborhood. The bars show the average values of the anisotropy variance. Only results for vector-based linear interpolation $(\square)$ and the proposed method $(\square)$ are shown since these two methods yield better structural clarity.

results, shown in Fig. 4, indicate that the proposed method, while giving markedly improved structural details as shown in the previous section, also yields greater structural smoothness. Such smoothness is important for applications such as tractography to ensure that fiber pathways can be traced more reliably with greater continuity. The blocking artifacts caused by vector-based linear interpolation will cause abrupt changes in local fiber orientations, the effect of which on tractography can be disastrous. 


\section{Conclusion}

We have presented a technique that increases the image resolution of DWI data beyond the acquired resolution, producing results that are free from blocking artifacts and imaging noise while at the same time show excellent structural clarity. Our approach produces spatially dense vector-valued data that are available for all diffusion gradient directions as well as all tissue types. This important distinction essentially implies that, with the results generated by our approach, the myriad of existing diffusion models can be fitted for multifaceted analysis. In addition, our work also prompts the need of a closer examination on the effect of interpolation artifacts on the performance of various DWI algorithms, such as tractography. Future work will be directed at utilizing locally non-linear interpolation strategies to elucidate even finer structural details and to resolve complex fiber architectures such as configurations associated with branching and kissing.

Acknowledgment. This work was supported in part by a UNC start-up fund and NIH grants (EB006733, EB008374, EB009634, MH088520, and AG041721).

\section{References}

1. Calamante, F., Tournier, J., Jackson, G., Connelly, A.: Track-density imaging (TDI): Super-resolution white matter imaging using whole-brain track-density mapping. Neuroimage 53(4), 1233-1243 (2010)

2. Jian, B., Vemuri, B.C.: A unified computational framework for deconvolution to reconstruct multiple fibers from diffusion weighted MRI. IEEE Transactions on Medical Imaging 26(11), 1464-1471 (2007)

3. Lee, H., Battle, A., Raina, R., Ng, A.Y.: Efficient sparse coding algorithms. In: NIPS, pp. 801-808 (2007)

4. Liu, C., Bammer, R., Kim, D.H., Moseley, M.E.: Self-navigated interleaved spiral (SNAILS): Application to high-resolution diffusion tensor imaging. Magnetic Resonance in Medicine 52(6), 1388-1396 (2004)

5. Liu, T., Li, H., Wong, K., Tarok, A., Guo, L., Wong, S.T.: Brain tissue segmentation based on DTI data. NeuroImage 38(1), 114-123 (2007)

6. Nedjati-Gilani, S., Alexander, D., Parker, G.: Regularized super-resolution for diffusion MRI. In: IEEE International Symposium on Biomedical Imaging: From Nano to Macro, pp. $875-878$ (2008)

7. Nowak, R.: Wavelet-based Rician noise removal for magnetic resonance imaging. IEEE Transactions on Image Processing 8(10), 1408-1419 (1999)

8. Scherrer, B., Gholipour, A., Warfield, S.K.: Super-Resolution in Diffusion-Weighted Imaging. In: Fichtinger, G., Martel, A., Peters, T. (eds.) MICCAI 2011, Part II. LNCS, vol. 6892, pp. 124-132. Springer, Heidelberg (2011) 